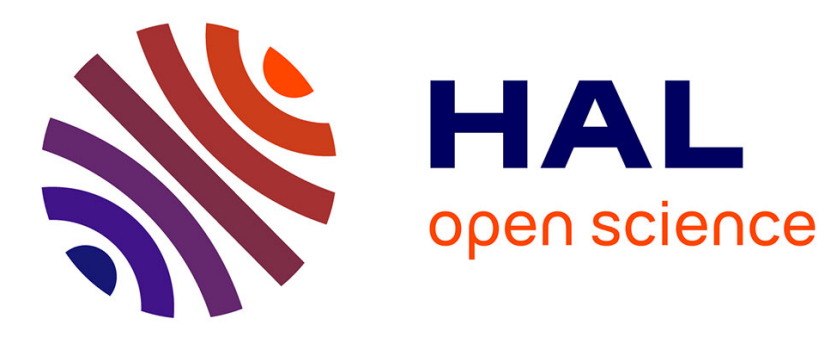

\title{
Time reversal kaleidoscope: A smart transducer for three-dimensional ultrasonic imaging
} Gabriel Montaldo, Delphine Palacio, Mickaël Tanter, Mathias Fink

\section{To cite this version:}

Gabriel Montaldo, Delphine Palacio, Mickaël Tanter, Mathias Fink. Time reversal kaleidoscope: A smart transducer for three-dimensional ultrasonic imaging. Applied Physics Letters, 2004, 84 (19), pp.3879-3881. 10.1063/1.1738186 . hal-02481900

\section{HAL Id: hal-02481900 https://hal.science/hal-02481900}

Submitted on 17 Feb 2020

HAL is a multi-disciplinary open access archive for the deposit and dissemination of scientific research documents, whether they are published or not. The documents may come from teaching and research institutions in France or abroad, or from public or private research centers.
L'archive ouverte pluridisciplinaire HAL, est destinée au dépôt et à la diffusion de documents scientifiques de niveau recherche, publiés ou non, émanant des établissements d'enseignement et de recherche français ou étrangers, des laboratoires publics ou privés. 


\section{Time reversal kaleidoscope: A smart transducer for three-dimensional ultrasonic imaging}

Cite as: Appl. Phys. Lett. 84, 3879 (2004); https://doi.org/10.1063/1.1738186

Submitted: 22 December 2003 . Accepted: 10 March 2004 . Published Online: 29 April 2004

Gabriel Montaldo, Delphine Palacio, Mickael Tanter, and Mathias Fink

\section{ARTICLES YOU MAY BE INTERESTED IN}

Acoustic imaging device with one transducer

The Journal of the Acoustical Society of America 131, EL395 (2012); https://

doi.org/10.1121/1.3699533

One-channel time-reversal in chaotic cavities: Experimental results

The Journal of the Acoustical Society of America 105, 618 (1999); https://

doi.org/10.1121/1.426252

In solid localization of finger impacts using acoustic time-reversal process

Applied Physics Letters 87, 204104 (2005); https://doi.org/10.1063/1.2130720

Lock-in Amplifiers Find out more today

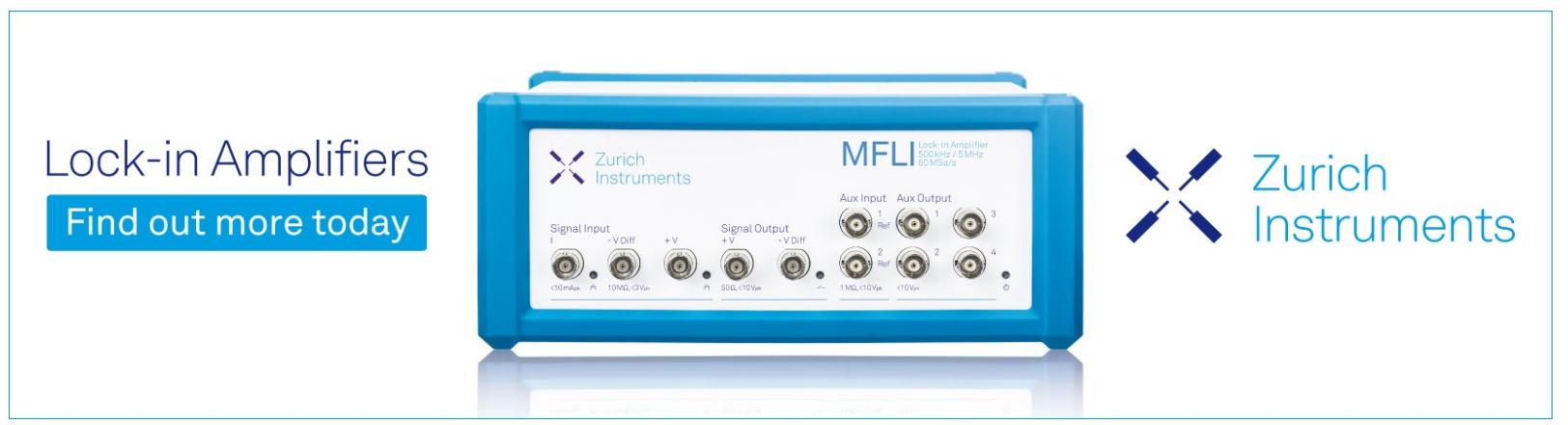




\title{
Time reversal kaleidoscope: A smart transducer for three-dimensional ultrasonic imaging
}

\author{
Gabriel Montaldo, Delphine Palacio, ${ }^{\text {a) }}$ Mickael Tanter, and Mathias Fink \\ Laboratoire Ondes et Acoustique, 10, rue Vauquelin, 75005 Paris, France
}

(Received 22 December 2003; accepted 10 March 2004; published online 29 April 2004)

\begin{abstract}
The design of two dimensional (2D) arrays for three dimensional (3D) ultrasonic imaging is a major challenge in medical and nondestructive applications. Thousands of transducers are typically needed for beam focusing and steering in 3D volumes. Here, we report a promising approach for producing 3D images with a small number of transducers using the combined concepts of time reversal mirrors and chaotic reverberating cavities. Due to multiple reverberations inside the cavity, a "kaleidoscopic" transducer array is created with thousands of virtual transducers equivalent to 2D matrices. Beyond the scope of 3D medical imaging, this work leads to the concept of "smart" transducer. (C) 2004 American Institute of Physics. [DOI: 10.1063/1.1738186]
\end{abstract}

A first generation of three-dimensional (3D) ultrasonic scanners have recently appeared in clinical environment, they are able to display the surfaces of some well contrasted interfaces like the face of a baby or the cavities of the heart. These systems combine series of successive 2D images produced by a conventional unidimentional array ${ }^{1}$ moved either by the practician or by a motorized device. A more sophisticated approach would consist in designing $2 \mathrm{D}$ arrays made of thousands of ultrasonic transducers. However, the problem of connecting such a huge number of elements independently has not been ovecome to date. Therefore, limited subapertures of the complete array can be driven simultaneously by the electronics through a complex multiplexing system. ${ }^{1,2}$

In this letter, we present an approach based on the concept of time reversal mirrors. Our goal is to propose a solution that combines the use of time reversal technology with a small number of piezoelectric transducers fastened to a reverberating solid cavity with one face in contact with the investigated medium. Time reversal focusing has been previously studied in waveguides in the field of ultrasound ${ }^{3}$ and in ocean acoustics. ${ }^{4}$ This technique is based on the reversibility of acoustic propagation ${ }^{5}$ which implies that the time-reversed version of an incident pressure field naturally refocuses in space and time on its source whatever the heterogeneity of the propagation medium. This idea which leads to the concept of time reversal pulse recompression in a reverberating medium has been successfully applied in solid waveguides for shock wave lithotripsy. ${ }^{6}$ In such configurations, one face of the waveguide was in contact with a patient body while a small quantity of transducers were glued on the opposite face. Thanks to the multiple reverberations on the waveguide boundaries, the waves emitted by each transducer are multiply reflected, creating at each reflection "virtual" transducers that can be observed from the desired focal point. Thus, we create a large virtual array from a limited number of transducers. The result of such an operation is that a small number of transducers is multiplied to create a "kaleidoscopic" transducer array. However, the symmetries implied in

${ }^{a)}$ Electronic mail: delphine.palacio@loa.espci.fr waveguides create periodic kaleidoscopic arrays resulting in grating lobes that limit the interest of this technique to shock wave generation for lithotripsy.

To extend this concept to pulse-echo imaging, sidelobes have to be strongly reduced. The solution we propose is to break waveguide symmetries by introducing reverberating media with chaotic geometries such as chaotic billiards. Preliminary work on time reversal in 2D closed cavities was done by Draeger et al. using Bunimovitch billiards. ${ }^{7}$ We extend this work to 3D leaky cavities and we select a Sinai billiard geometry to achieve 3D focusing.

A first 3D Sinai billiard has been designed in duraluminum $\left(50 \times 50 \times 50 \mathrm{~mm}^{3}\right)$. Thirty transmit single elements $\left(0.8 \times 0.5 \mathrm{~mm}^{2}\right.$ rectangular piezoelectric ceramics, $1.5 \mathrm{MHz}$ central frequency) have been glued on one face of the billiard. The bottom face has been placed in contact with a fluid medium (see Fig. 1). Every transducer has been connected to a fully programmable transmit/receive multichannel electronic system (32 MHz sampling frequency, $80 \mathrm{~V}_{p p} / 50 \Omega$ ).

In order to focus a pulse in any point of the fluid, a time reversal process is implemented. In a first step, an ultrasonic source located at the desired focusing location emits a short

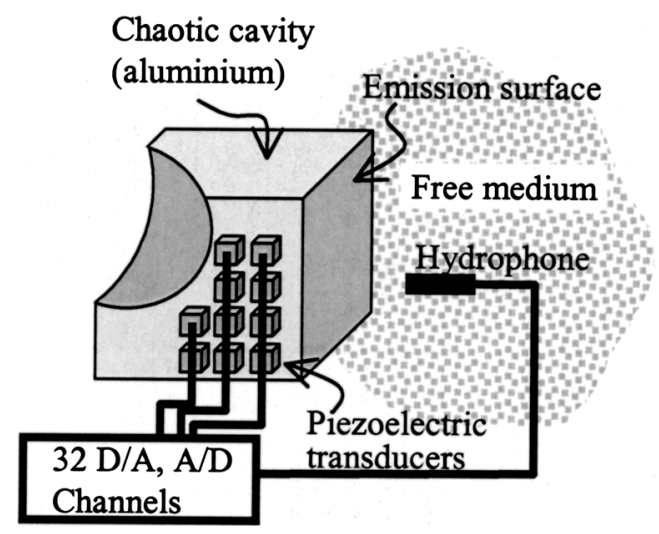

FIG. 1. Experimental setup. The kaleidoscope is composed by a chaotic cavity in duraluminum and 30 piezoelectric transducers $\left(0.5 \times 0.8 \mathrm{~mm}^{2}\right)$ glued on one face. Each transducer is connected to an independent digitalto-analog and analog-to-digital channel. One surface of the kaleidoscope is in contact with the imaged medium. 

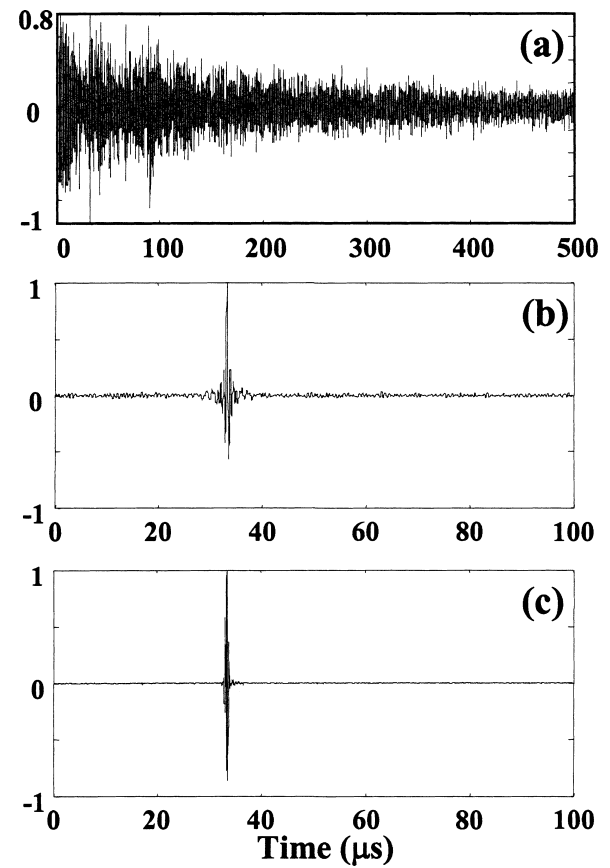

FIG. 2. (a) Signal registered by a transducer of the kaleidoscope after sending an impulsion of $2 \mu \mathrm{s}$ from a hydrophone. Due to the multiple reflections on cavity faces, the response is very long (up to $500 \mu \mathrm{s}$ ). (b) time-reversal recompression $(2 \mu \mathrm{s})$ received by the hydrophone, the sidelobes have a $-38 \mathrm{~dB}$ level; (c) first harmonic pulse on the hydrophone; the sidelobes have $-60 \mathrm{~dB}$.

pulse (typically, $1 \mu$ s duration). After propagation in the fluid, the resulting wavefront enters the cavity. Due to the strong reverberations inside the cavity, waves are reflected hundreds of times and the impulse responses received by each transducer of the cavity are strongly spread in time (typically, up to $500 \mu \mathrm{s}$ ). In a second step, the signals are
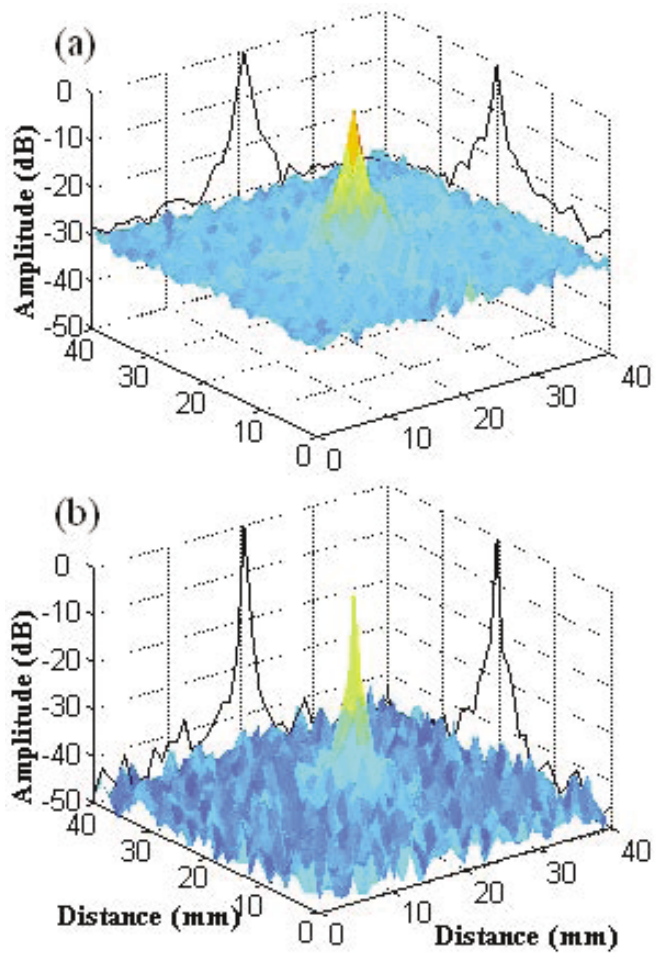

FIG. 3. (a) Spatial quality of the focusing in a plane orthogonal to the beam axis using the fundamental frequency the sidelobes level of the fundamental pulse is $-30 \mathrm{~dB}$; (b) same than (a) but using the first harmonic the level of the first harmonic pulse is $-50 \mathrm{~dB}$; (c) focus on three different points (pitch $10 \mathrm{~mm}$ ): similar spatial pattern for the fundamental pulse and the first harmonic pulse; (d) 3D images of a phantom object obtained with the kaleidoscope using 30 emitters and only one omnidirectionnal receiver. time reversed and reemitted by the individual elements. Due to time reversal symmetry, waves refocus at the initial source location both in space and time as if time was playing backwards. Note, that because of spatial reciprocity, the first step can be achieved in another way. The impulse responses can be successively acquired by emitting a short pulse from each individual transducer and recording the resulting signals with a hydrophone at the desired focus in the fluid.

For each focal point in the fluid, a data set of 30 impulse responses can be acquired and stored in memories. An example of such "coda" waveforms is shown in Fig. 2(a) for a desired focal spot located at $40 \mathrm{~mm}$ of depth. It corresponds to nearly $1.5 \mathrm{~m}$ reverberant propagation in duraluminum equivalent to nearly 300 reflections on the boundaries. Figure 2(b). presents the signal received at the focus after time reversal and reemission of the 30 coded waveforms. We note a very good temporal recompression of the signal, however, temporal sidelobes remain with a $-38 \mathrm{~dB}$ level. The hydrophone can also be used to scan the time reversed wavefield around the focus. Figure 3(a) shows the spatial directivity pattern of the maximal pressure field in a plane orthogonal to the beam axis. The $-6 \mathrm{~dB}$ width is equal to $1.5 \mathrm{~mm}$ corresponding to a total transmit aperture of $33 \times 33 \mathrm{~mm}^{2}$. Spatial sidelobes are also seen in Fig. 3(a). and reach a $-30 \mathrm{~dB}$ level.

This focusing quality in the transmit mode is good and could provide 3D echographic images with a good contrast if we were also able to apply the same focusing concept in the receive mode. In the transmit focusing mode all the coda energy sent from the solid are recompressed in a high amplitude pulse by the time reversal process however, the receive focusing mode is not very efficient. Indeed, the signals com-
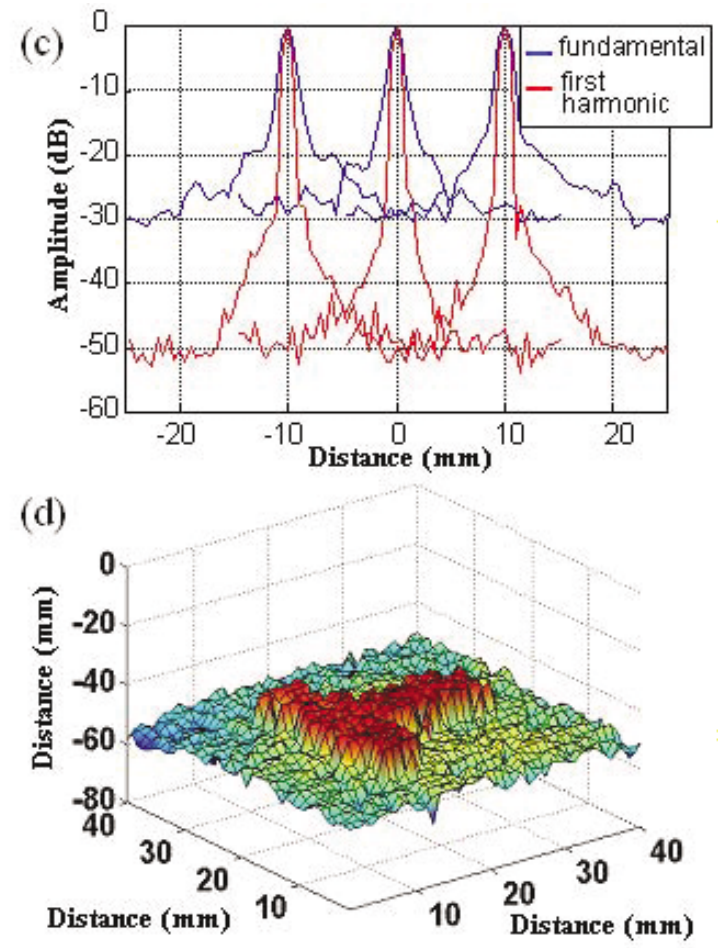
ing back from a target are weak and almost totally reflected at the front face of the chaotic cavity giving rise to very small signals on the transducers. Another drawback comes from the fact that these echoes are superimposed to the remaining reverberations of the incident wave.

In this letter, we present an elegant solution to this problem by exploiting the nonlinear propagation of the focused beam in the fluid. As the time reversed pulse reaches a very intense amplitude around the focus, nonlinear effects appear in the fluid which give rise to strong harmonic generation. As the harmonic sources depend on the square of the incident pressure field amplitude, ${ }^{8}$ the directivity pattern of the first harmonic should be cleaned of spurous signals. Figures 2(c) and 3(b) show, respectively, the first harmonic pulse received at the focus and its spatial directivity pattern. These data are obtained by filtering the first harmonic from the fundamental component using an analogic highpass filter. In Fig. 2(c), the temporal sidelobe level has been strongly reduced up to $-60 \mathrm{~dB}$ while the spatial pattern in Fig. 3(b). exhibits a $-50 \mathrm{~dB}$ sidelobe level.

The ability of this system to focus in a large 3D volume is illustrated in Fig. 3(c). The spatial directivity patterns along an axis orthogonal to the beam axis are presented for both fundamental and harmonics while emitting three different sets of signals adapted to three different locations. Contrast improvement with harmonic filtering is maintained on the whole field of interest.

In order to perform an harmonic image of the investigated medium we first need to calibrate our time reversal kaleidoscope in a reference homogeneous medium (water) by recording the data sets of emission signals corresponding to a complete $3 \mathrm{D}$ volume of focal spots. The technique is then used to focus sequentially on each spot in the region of interest (for medical applications, in the patient body). As the biological tissues have nearly the same impedance as water, the kaleidoscope does not see any change and performs the same focusing as in water. The backscattered echoes are recorded by one or several reception transducers stuck on the front face of the kaleidoscope and centered in the harmonic frequency. Figure 3(d) shows a 3D image of the surface of a gelatin phantom in shape of " $\mathrm{T}$ " obtained with the 30 transmit transducers kaleidoscope using a single omnidirectionnal receiving element.

The time we need to build an image is limited by the reverberation time in the cavity. To focus a pulse in a point the acoustical signal reverberates nearly $500 \mu \mathrm{s}$, then, to build a complete image in a grid of 40 by 40 points we need $0.8 \mathrm{~s}$. This is very slow for a real-time visualization. A simple solution to increase the frame rate is to overlap the emissions of different points. For example, if $s_{1}(t)$ is the code to focus in point $1, s_{2}(t)$ to focus in point 2 and so on, we can send a signal $s_{1}(t)+s_{2}(t+\Delta)+s_{3}(t+2 \Delta)$, then, we obtain a sequence of pulses separated by a time $\Delta$ that focus in points 1 , 2 , and 3. Using a separation time of $150 \mu$ s the total time of the same image is $0.24 \mathrm{~s}$.

This concept of smart transducers can be extended by using more transducers in the receive mode and a prototype combining 64 transmit and 64 receive elements is currently under construction. Contrary to 2D matrices technology, the system complexity is transferred from connectivity to the more suitable concept of emissions coda stored in memories. This technique has a strong potential for pulse echo medical imaging as well as for 3D shear source generation in soft tissues activated by the ultrasonic radiation force. The potential of such "smart" transducers is also envisioned for ultrasonic therapy.

${ }^{1}$ T. R. Nelson and D. H. Pretorius, Ultrasound Med. Biol. 24, 1243 (1998).

${ }^{2}$ E. D. Light, R. E. Davidsen, J. O. Fiering, T. A. Hruschka, and S. W. Smith, Ultrason. Imaging 20, 235 (1998).

${ }^{3}$ P. Roux, B. Roman, and M. Fink, Appl. Phys. Lett. 70, 1811 (1997).

${ }^{4}$ W. A. Kuperman, W. S. Hodgkiss, H. C. Song, T. Akal, C. Ferla, and D. R. Jackson, J. Acoust. Soc. Am. 103, 25 (1998).

${ }^{5}$ M. Fink, Phys. Today 50, 34 (1997).

${ }^{6}$ G. Montaldo, P. Roux, A. Derode, C. Negreira, and M. Fink, Appl. Phys. Lett. 80, 897 (2002).

${ }^{7}$ C. Draeger and M. Fink, Phys. Rev. Lett. 79, 407 (1997).

${ }^{8}$ M. F. Hamilton and D. T. Blackstock, Nonlinear Acoustics (Academic, New York, 1998). 